\title{
Design and Implementation of an Intelligent Tourist Guide System
}

\author{
Xinling Wang ${ }^{\mathrm{a},{ }^{*}}$ and Chengzhang Han $^{\mathrm{b}}$ \\ Qingdao Binhai University, Qingdao 266555, China \\ aemail:447991452@qq.com, bemail:420758217@qq.com, "corresponding author
}

Keywords: Intelligent Tourist Guide (ITG); GIS; ARM11; Handheld terminal

\begin{abstract}
Aiming at the shortcomings of Traditional Guide and popularization in the existing Intelligent Tourist Guide (ITG) system, this paper designs an ITG system named iGuider (intelligent-Guider), which is based on user-centered design principles and in view of usability and user experience perspective. iGuider uses the ARM11 processor and embedded systems, integrates Geographic Information System (GIS), multichannel interaction technologies and RFID to provide multimedia attraction information for tourists. Test result indicates that iGuider is reliable, energy efficient, good user experience, and has good prospects in tourism market.
\end{abstract}

\section{Introduction}

As a new economic growth point, the tourism industry has drawn increasing attention to the various national authorities, and convenient information service for sightseeing are urgently needed. There are several methods for quick and easy dissemination service information for sightseeing, such as Web online information, portable tour guiding speaker, site map, and so on [1]. However, as a new carrier of information dissemination, the intelligent tourist guide system has been leading the tourist industry to an electronic and intelligent direction. Since the first ITG prototype arose in the middle of the 1990s, the process of the ITG development can be divided into three stages: The first stage could only show simple maps with black and white, provide the basic positioning services, and was in the experimental research mostly. The second stage could combine two-dimensional maps and three-dimensional graphics for mobile systems, and could be run on mobile phones, such as TellMaris mobile guide system prototype developed by Nokia Research Center [2]. The third stage tends to be a humane and intelligent portable instrument guide [3][4], such as Gullivers Gunie [5], which is a prototype from the fields of artificial intelligence and agent system, can provide better contextual information according to user needs and location. The system can automatically display the media associated with the attraction during user enters a certain location,. These forms of media include, but are not limited to text, pictures, video, and other forms of multimedia.

Based on user-centered design principles, this paper designs the iGuider (intelligent-Guider) system from the point of humanization and intelligence, which is based on ARM11 and embedded systems [6][7], integrates Geographic Information System (GIS), RF modules [3], multichannel interaction and other modules. The system uses digital vector maps to provide real-time attractions routes, provides tourists attractions with multimedia information through information technology, automatically play information with appropriate language and content depending on the position and the need of the tourist, extends a variety of languages to meet the needs for tourists of different nationalities. The system has changed the previous manual "guided tour" mode, and becomes intelligent with good features as automatic recognition, automatic playback, multilingual, free choice, and also has many characteristics including intelligent, personalized, high-quality, stable, practical, etc..

\section{Portable Intelligent Gide System}

The overall structure of the system is shown in Figure1, which can be divided into two parts of Positioning module and handheld terminal. The RF transmitter module is installed at each spot or stand, and periodically launches its own address code. Different spots have different address codes. After visitors reach the scenic spot, the handheld terminal will automatically receive the coded 
signal. Through the analysis of the address code, the receiving terminal calls and switches to the address code corresponding to the interpretation of the file playback, and displays the attractions photos or videos on the screen at the same time.

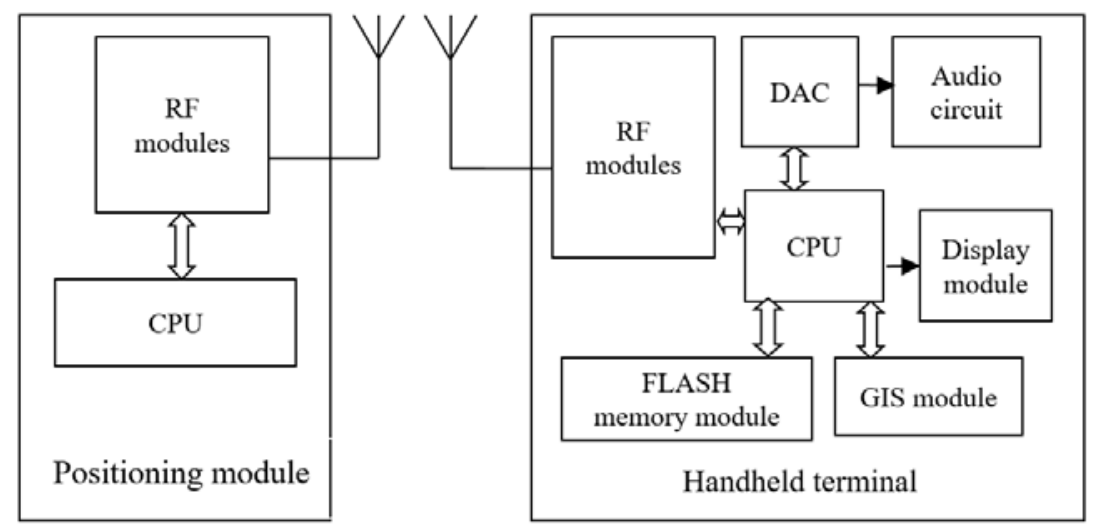

Fig.1. Illustrative view of the iGuider system

Positioning Module. The positioning module and the hand-held terminal both have a radio frequency module, which is responsible for transmitting and receiving ID respectively. The system chooses the CC2500 chip and PIC16F636 to form the electronic label. The CC2500 chip is a single-chip wireless transceiver integration chip introduced by the Chipcon company, working in 2.4 GHz ISM (industrial, scientific, medical) band, meeting needs for multipoint communication and frequency hopping communication, operating voltage of $1.9-3.6 \mathrm{~V}$, which also has such characteristics as low power consumption, peripheral elements rarely, built-in hardware CRC (cyclic redundancy check) and point to multipoint communication address control, etc.. The chip integrates almost all of the RF transceiver module, including the programmable phase synchronization logic synthesizer (PLL), RF power amplifier (PA), low noise amplifier (LNA), baseband filter and amplifier and I/Q demodulator. The main working parameters can be configured by the user according to the needs of the chip state, which have no complex communication protocol and easy to achieve ID code transceiver.

Control Module. In the Control module, the core processor will be powered by an ARM11 core S3C6410 processor, with the operating system running the embedded Linux operating system. S3C6410 is a high performance and low power RSIC general purpose microprocessor based on 16/32 ARM1176, which is suitable for handheld, mobile and other terminal devices. It is a low-power, cost-effective, high-performance for mobile phones and general processing RSIC processor, provides optimized hardware performance for 2.5G and 3G communication services, and uses 64/32bit internal bus architecture with the integration of the AXI, AHB, APB bus. There are many powerful hardware accelerators, including motion video processing, audio processing, 2D acceleration, display processing and scaling. An integrated MFC (Multi-Format Video Codec) supports decoding MPEG4 / H.263 / H.264 and VC1 decoding. This hardware codec supports real-time video conferencing as well as NTSC and PAL TV output. The processor is highly integrated, powerful, suitable for portable devices and other industrial equipment, such as a fingerprint reader, and onboard systems.

GIS Module. This module handles the user's control information, including map roaming, zoom, query function (as shown in Figure 2 below) and guide function, such as pen click information explaining, tourist facilities searching, and a map database accessing. Dynamic path analysis and the shortest path function and other advanced services should also be completed in conjunction with this module. 


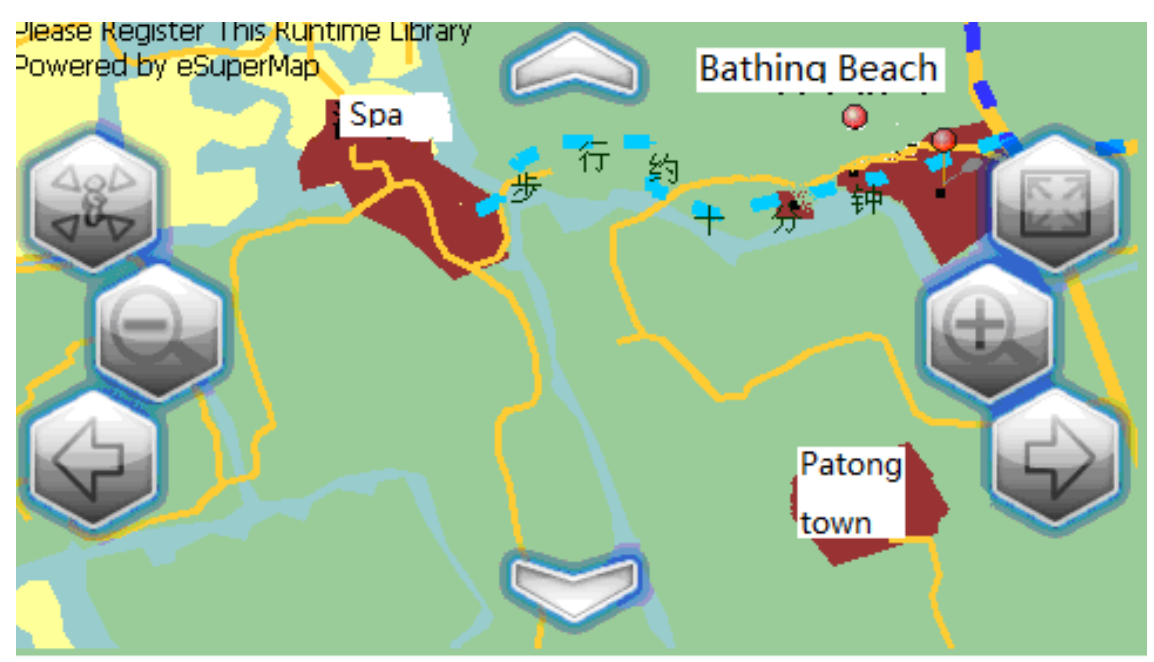

Fig.2. Electronic map operation interface

Display Module. The display module has two parts consist of visual display and auditory display. The visual display provides rich video information. Auditory display contains attraction commentary, path tips, public facilities reminders, ad player, sound reminders, etc. This module provides a wealth of multimedia information; the user can choose how the information is presented according to personal preference. Figure 3 is a multimedia user interface.

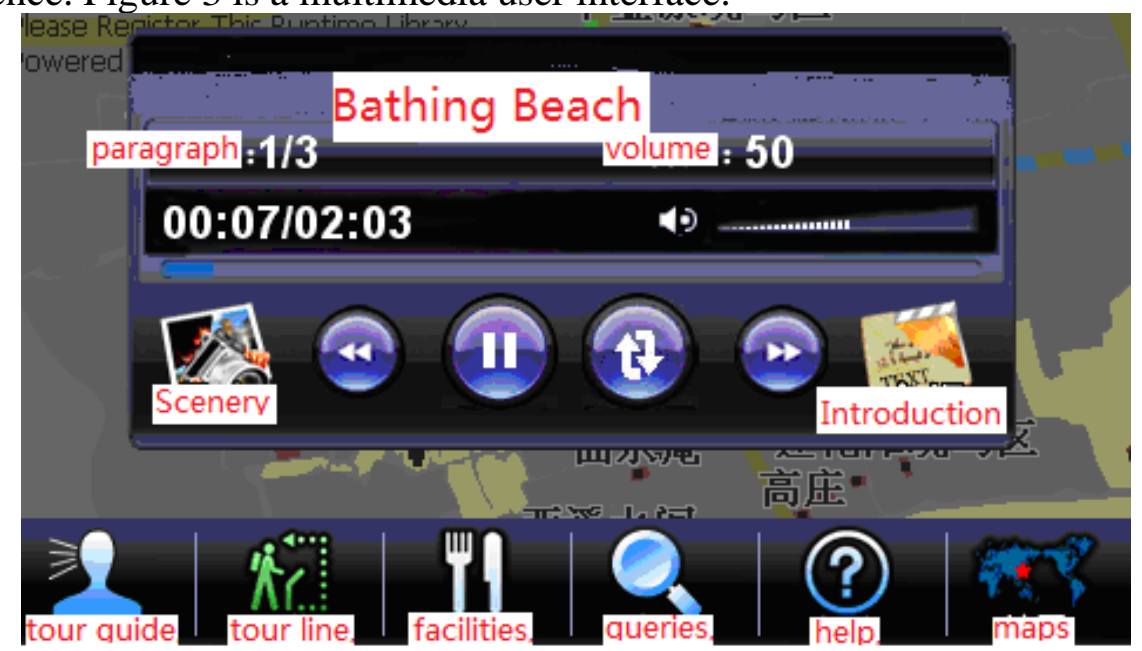

Fig.3. The multimedia user interface

\section{Software Protocol Implementations}

Communications between positioning module and handheld terminal will be achieved by positioning module in point to multipoint wireless communication mode. The common point to multipoint communication system uses two models of multi ring and star type. The ring system is mainly used for long transmission distance and not high real-time requirements of the occasion. The star system is especially suitable for the occasions where the data quantity is large and the real-time requirement is high. The wireless communication is adopted between the electronic label of positioning module in each attraction and the tour guide, and the guide system adopts the point (RF label) to the multi point (handheld terminal) star pattern.

The positioning module periodically launches its own address code to the outside, and different places have different address codes, though the same spot can have one or more label codes of the same address code. After tourists reach the scenic spot, the tourist guide on hand automatically receives the coded signal, and the control center can control the voice and the image to play after decoding.

In the field of wireless communication technology, different communication systems have different communication protocols. Protocol is used to identify noise and valid data. Noise has no obvious combination and appears in random bytes, and the noise source may produce any 
combination of bytes. In wireless communication, the effective protocol can restrain the noise generation. Through the test and the experiment, it turned out that 0XFF followed by 0X55, 0XAA in the noise is not easy to produce. Transmission protocol should be added before the start byte packet, and the protocol packet format is shown in Table 1.

Table 1 protocol packet format

\begin{tabular}{|c|c|c|c|c|c|c|c|c|}
\hline Random & Lead1 & Lead2 & Lead3 & Address & Data1 & Data1 & $\ldots$ & Checksum \\
\hline & 0xFF & 0xAA & 0x55 & & & & $\ldots$ & \\
\hline
\end{tabular}

Under the agreement, guides program flow chart can be written, which is shown in Figure 4.

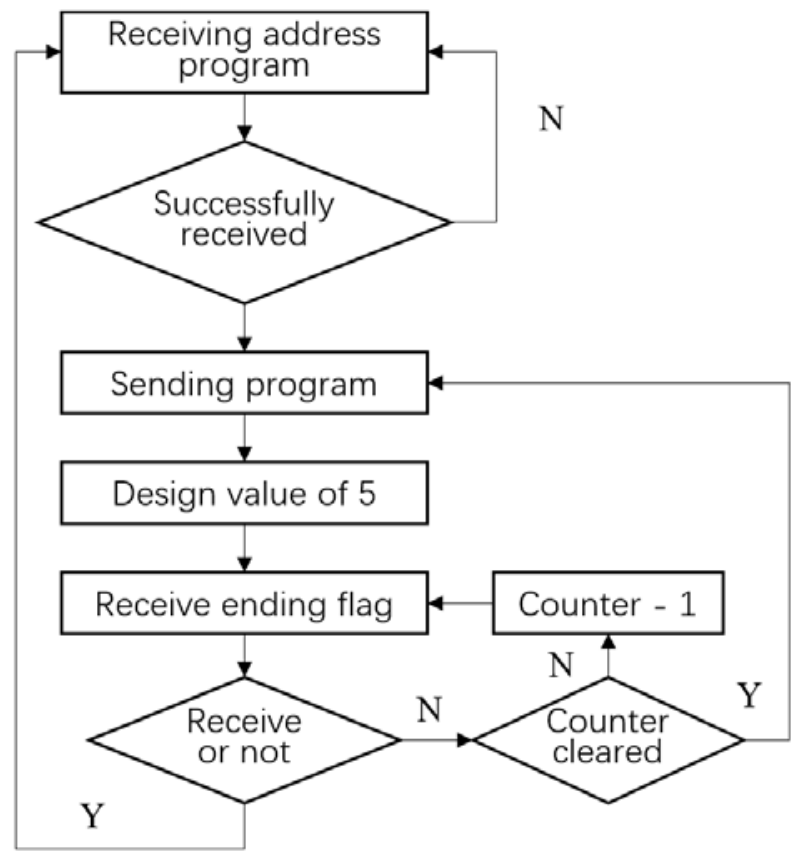

Fig.4. Program flow chart of tour guide

\section{Portable Handheld Guide}

In the apparent form, the handheld terminal is generally similar to the MP4, which is shown in Figure 5. When a tourist carries an intelligent terminal to visit a attractions in the scenic spot, the handheld terminal will match the electronic label of the attractions. After the success of the match, the microprocessor decodes and calls the corresponding speech and image files stored in the Flash.

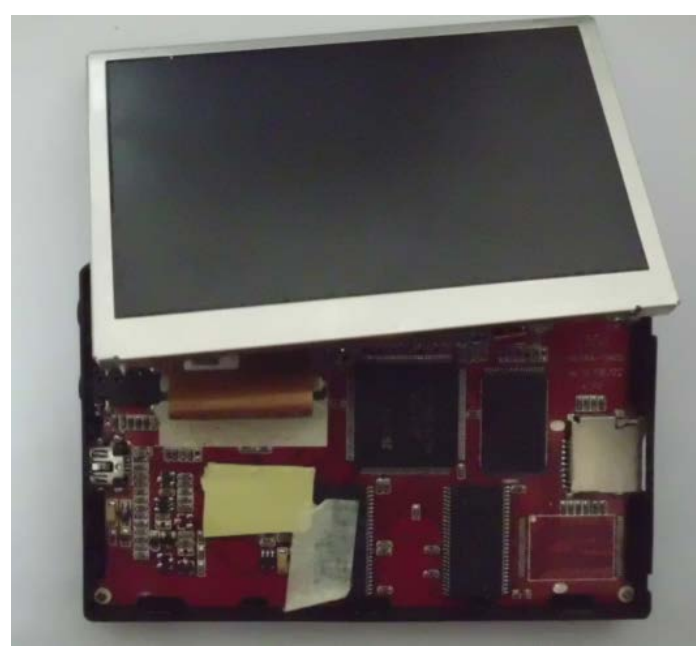

Fig.5. The preliminary physical map of handheld guide instrument 


\section{Summary}

Through the test of tourist attractions, results shows that the iGuider system is reliable, and easy to use. Be attributable to natural interface and good interaction design, users do not need long time to learn how to use the system. In addition, the system also has several merits such as low power consumption, rapid responses and good user experience, and so on. Predictably, once the iGuider system is putting on the tourism market, it will bring good economic and social benefits.

\section{References}

[1] Smirnov A, Kashevnik A, Balandin S I, et al. "Intelligent Mobile Tourist Guide,” in Internet of Things, Smart Spaces, and Next Generation Networking. Springer Berlin Heidelberg, 2013.94-106.

[2] Schilling A, Giersich M, Aasgaard R. "Introducing 3D GIS for the Mobile Community Technical Aspects in the Case of TellMaris,” International Workshop on Mobile Computing (IMC 2003), Fraunhofer IRB Verlag, April 2003.86-92.

[3] Owaied H H, Farhan H A, Alhawamdeh N, et al. A model for intelligent tourism guide system [J]. Journal of Applied Sciences, vol. 2011 11(2) 342-347.

[4] Zhao Ji, Zhang Xuedong. Design and Implementation of a Handheld Electronic Tour Guide System [J].Journal of University of Science and Technology Liaoning. vol. 2006 29(4) 381-384.

[5] O’Grady M J, O’Hare G M P. Gulliver's Genie. Agency, Mobility\&Adaptivity. Journal of Computers \& Graphics, vol. 2004 28(4) 677-689.

[6] Yuan Minjie, Lv Baiquan. Intelligent Electronic Guide System Based on AT85C51SND3 and CC2500 [J]. Journal of Industrial Control Computer, vol. 2008 21(7) 74-75.

[7] Zhang Lun, Cai Lina. Intelligent Guide Instrument Based on ARM9 Processor[J].Electronic Component \& Device Applications, vol. 2008. 10(1) 18-20. 